\title{
Cobertura vacinal e fatores associados ao esquema vacinal básico incompleto aos 12 meses de idade, São Luís, Maranhão, Brasil, 2006
}

\author{
Vaccination coverage and factors associated \\ with incomplete basic vaccination schedule \\ in 12-month-old children, São Luís, \\ Maranhão State, Brazil, 2006
}

\section{Cobertura de vacunación y factores asociados con esquemas incompletos de vacunación básica a los 12 meses de edad, São Luis, Maranhão, Brasil, 2006}

\author{
Ana Valéria Carvalho Pires Yokokura ${ }^{1}$ \\ Antônio Augusto Moura da Silva 1 \\ Ariane Cristina Ferreira Bernardes 1 \\ Fernando Lamy Filho 1 \\ Maria Tereza Seabra Soares de Brito Alves 1 \\ Nayra Anielly Lima Cabra 1 \\ Rosângela Fernandes Lucena Batista Alves 1
}

\footnotetext{
${ }_{1}$ Departamento de Saúde Pública, Universidade Federal do Maranhão, São Luís, Brasil.

Correspondência A. V. C. P. Yokokura Pós-graduação em Saúde Coletiva, Departamento de Saúde Pública, Universidade Federal do Maranhão. Av. 02, Qd. 03, C. 15, São Luís, MA 65050-140, Brasil.

valeria.yokokura@hotmail.com
}

\begin{abstract}
The study aimed to evaluate vaccination coverage and factors associated with incomplete basic vaccination schedule at 12 months of age in 427 children aged 12-59 months in São Luis, Maranhão State, Brazil, 2006. This cross-sectional, population-based household survey used complex cluster sampling. Poisson regression with robust adjustment of variance was applied. Complete basic vaccination coverage was $71.9 \%$ for applied doses, $61.8 \%$ for valid doses, and $23.6 \%$ for correct doses. Hepatitis $B$ and tetravalent vaccines showed higher percentages of doses on dates or at intervals lower than recommended. Percentages of delayed vaccination were high, except for BCG. Incomplete basic vaccination was more frequent in girls and children from low-income and black families. Racial, gender, and socioeconomic factors posed barriers to complete vaccination, thus emphasizing the need for policies to address such inequalities.
\end{abstract}

Immunization; Immunization Coverage; Child Health

\section{Resumo}

O estudo objetivou avaliar a cobertura vacinal e os fatores associados ao esquema vacinal básico incompleto aos 12 meses de idade, em 427 crianças de 12 a 59 meses, em São Luís, Maranhão, Brasil, 2006. Trata-se de inquérito domiciliar transversal, de base populacional, com amostragem complexa por conglomerados. Empregouse regressão de Poisson com ajuste robusto da variância. A cobertura para o esquema básico completo foi de 71,9\% segundo doses aplicadas, $61,8 \%$ para doses válidas e $23,6 \%$ para doses corretas. As vacinas contra hepatite $B$ e tetravalente apresentaram maiores percentuais de doses aplicadas em datas ou intervalos inferiores aos recomendados. Os percentuais de atraso vacinal foram elevados, exceto para a BCG. Percentuais mais elevados de esquema vacinal básico incompleto foram encontrados em crianças das classes econômicas $D$ e E, do sexo feminino e de chefes de família de cor da pele preta. As desigualdades raciais, de gênero e socioeconômicas representaram barreiras à vacinação completa, indicando necessidade de reforçar as políticas equitativas que eliminem essas desigualdades.

Imunização; Cobertura Vacinal; Saúde da Criança 


\section{Introdução}

A Organização Mundial da Saúde (OMS) e o Fundo das Nações Unidas para a Infância (UNICEF) 1 estimam que, a cada ano, morrem, no mundo, 2,5 milhões de crianças menores de cinco anos de idade por doenças que poderiam ter sido evitadas por ações de imunização. As vacinas, que salvam vidas, continuam inacessíveis a cerca de 24 milhões de crianças que estão expostas a um risco maior de adoecimento e morte 2 .

A vacinação é considerada uma das maiores conquistas em saúde pública do século XX e um dos três marcos mais importantes da saúde pública para prevenção e controle das doenças transmissíveis, sendo uma das medidas de prevenção mais seguras e de melhor relação custoefetividade para os sistemas de saúde ${ }^{3}$.

Por meio dela, já se alcançaram muitas conquistas. Dentre essas, destacam-se: a erradicação da febre amarela urbana, da varíola e da poliomielite, o controle do sarampo, do tétano neonatal e acidental, das formas graves de tuberculose, difteria e coqueluche. Consequentemente, a vacinação tem contribuído para a redução significativa das taxas de morbimortalidade infantil. Isso demonstra a trajetória excepcional do programa de imunização no âmbito das políticas de saúde no Brasil 4.

Apesar do declínio significativo das doenças imunopreveníveis no Brasil, são observados uma heterogeneidade acentuada na cobertura vacinal e um número considerável de crianças menores de um ano não vacinadas, o que representa risco para a introdução e manutenção da circulação de agentes infecciosos, requerendo estratégias distintas de vacinação 4,5,6.

Alguns fatores têm sido apontados na literatura como obstáculos para a vacinação, tais como: baixa renda, residência em área rural, extremos de idade materna, maior número de filhos, maior ordem de nascimento, baixa escolaridade materna, trabalho materno fora do lar, maior número de moradores no domicílio, residência há menos de um ano na área, falta de conhecimento acerca das doenças imunopreveníveis, ausência de seguro-saúde e presença de doença na criança 7,8,9.

A identificação das crianças suscetíveis à não vacinação e o levantamento de informações precisas e atuais sobre as barreiras que dificultam a implementação das metas do Programa de Imunização tornam-se um elemento fundamental. Portanto, estimativas precisas de cobertura vacinal, obtidas por meio de inquéritos domiciliares, constituem instrumentos fundamentais para a adequada monitorização dos programas de vacinação e para se identificar e atingir as crianças que não são vacinadas, permitindo avaliar disparidades inter e intrarregionais, identificar e corrigir as desigualdades sociais existentes 7,10,11. É importante também se estimar as coberturas vacinais considerando-se não somente as doses aplicadas, mas segundo os critérios de doses válidas e corretas, respeitando-se as idades recomendadas e intervalos mínimos entre as doses. Entretanto, poucos têm sido os trabalhos que estimam a cobertura vacinal utilizando critérios mais rigorosos de doses válidas e corretas 9,11.

Diante do exposto, o objetivo deste estudo foi avaliar a cobertura vacinal e investigar a associação entre esquema vacinal básico incompleto e fatores socioeconômicos e demográficos aos 12 meses de idade.

\section{Métodos}

Trata-se de estudo transversal, de base populacional, por inquérito domiciliar, que é parte integrante da pesquisa Atenção à Saúde no Estado do Maranhão, realizada em 2006-2007 12. A população de estudo foi composta por crianças de 1259 meses, residentes no Município de São Luís, Maranhão, Brasil.

Foi realizada amostragem probabilística por conglomerados em dois estágios de seleção. No primeiro, foram amostrados 58 setores censitários, com probabilidade proporcional à população, a partir do censo de 2000. Foram sorteados o quarteirão inicial dentro de cada setor e um ponto inicial dentro de cada quarteirão, percorrido em sentido horário até que se entrevistassem 12 crianças em cada setor, não se limitando o número de crianças incluídas por domicílio. Nos casos em que esse número de crianças não foi encontrado, foi sorteado outro quarteirão dentro do mesmo setor até se atingir o número desejado de crianças.

No segundo estágio, a unidade de amostragem foi a criança, mas o processo de amostragem foi realizado por domicílio. Se na última casa morassem mais crianças do que o necessário para se completar 12 por setor, todas as crianças eram incluídas, e, nesses casos, foram incluídas mais de 12 por setor. Como os domicílios foram selecionados com aproximadamente a mesma probabilidade, não houve necessidade de ponderação.

Estimou-se uma amostra de 448 crianças, tendo, como base, uma estimativa de cobertura do esquema vacinal básico incompleto de $30 \%$, com nível de 95\% de confiança, assumindo-se população infinita, precisão da estimativa de $6 \%$ e efeito de desenho igual a 2,0. Para esse cálculo, 
foi utilizada a fórmula $n=\left(\mathrm{z}^{2} \times \mathrm{p} \mathrm{x} \mathrm{q}\right) / \mathrm{d}^{2}$, com correção para o efeito de desenho.

A amostra selecionada foi composta por 547 crianças. Desse conjunto, foram excluídas 7 por apresentarem inconsistências entre a data de nascimento e as datas de vacinação. Não foi possível calcular a proporção de esquema vacinal incompleto para 113 crianças, pois não foi apresentado cartão de vacinas no momento da entrevista, o que representou perda de $21 \%$, resultando em amostra final de 427 crianças.

Utilizou-se questionário padronizado, respondido pela mãe ou responsável pela criança. As informações sobre vacinação foram obtidas diretamente dos cartões de vacina. Foi calculada a cobertura vacinal a partir do percentual de crianças que realizaram as doses de cada uma das vacinas e o esquema básico completo aos 12 meses, considerando-se o calendário básico do primeiro ano de vida preconizado pelo Programa Nacional de Imunizações 13: uma dose de BCG, três doses de DPT-Hib, três doses de poliomielite e três doses de hepatite B. Empregaram-se os critérios do Ministério da Saúde 10,14 para a definição de doses inválidas e atrasadas, considerando-se a idade e o intervalo mínimo entre as doses. Considerou-se dose atrasada quando administrada após 30 dias da data agendada. $\mathrm{O}$ cumprimento do esquema básico completo levou, em consideração, as seguintes definições: (a) esquema básico completo por doses aplicadas: doses de cada uma das vacinas com aplicação prevista para o primeiro ano de vida, sem levar, em consideração, a idade e o intervalo mínimo entre as doses; (b) esquema básico completo por doses válidas: doses de cada uma das vacinas com aplicação prevista para o primeiro ano de vida, considerando-se a idade e o intervalo mínimo entre as doses; e (c) esquema básico completo por doses corretas: doses de cada uma das vacinas com aplicação prevista para o primeiro ano de vida, considerando-se a idade e o intervalo correto entre as doses.

A vacina tetravalente $(\mathrm{DTP}+\mathrm{Hib})$ passou a integrar o calendário apenas no ano de 2002, e algumas crianças entrevistadas foram vacinadas previamente a essa data, recebendo apenas a vacina tríplice bacteriana (DTP) ou as duas vacinas de forma não combinada. Por esse motivo, as crianças que receberam apenas DTP ou DTP+Hib não tiveram distinção na marcação do item tetravalente ou DTP.

A variável desfecho foi o esquema vacinal básico incompleto. As variáveis independentes relativas aos chefes de família foram: sexo; idade ( $<30$ anos, 30-39 anos, 40-49 anos, $\geq 50$ anos); escolaridade ( $\leq 4$ anos, 5-8 anos, 9-11 anos, $\geq 12$ anos); classificação econômica (Critério de Clas- sificação Econômica Brasil) segundo a Associação Brasileira de Empresas de Pesquisa - ABEP (A, B, C, D, E); renda familiar em salários mínimos (até $1,>1$ até $3,>3$ até $5,>5$ ); situação conjugal (com ou sem companheiro); inserção na população economicamente ativa (inserido ou fora da população economicamente ativa) e cor da pele autorreferida (branca, parda, preta).

A classificação econômica da ABEP é construída a partir de um escore que leva, em consideração, a posse de bens de consumo (televisão, geladeira, rádio, automóvel, empregada doméstica, máquina de lavar, videocassete e freezer) e a escolaridade do chefe de família. Classifica-se em A, B, C, D e E, sendo que a classe "A" referese à classe econômica mais alta, e a "E", à classe mais baixa.

Além dessas variáveis, foram utilizados dados da criança: sexo, idade (1-2 anos, 3-4 anos), cor da pele referida pela mãe ou responsável (branca, preta e parda), número de irmãos (até 2, 3 ou mais), idade da mãe ( $<20,20-34, \geq 35$ anos), recebimento de benefício de programas de transferência de renda do governo, principalmente Bolsa Família, cadastramento no PSF (Programa Saúde da Família) e posse de seguro-saúde.

As análises estatísticas foram realizadas no pacote estatístico Stata, na versão 9.0 (Stata Corp., College Station, Estados Unidos), utilizando-se o conjunto de comandos svy. O uso de estimadores convencionais poderia subestimar consideravelmente a variância das estimativas pontuais 15 . O conjunto de comandos svy levou, em consideração, o delineamento amostral complexo.

A probabilidade de a criança ser sorteada em cada estágio foi calculada. No primeiro estágio, essa probabilidade foi obtida multiplicando-se o número de setores amostrados no município pelo número de crianças de 12-59 meses no setor censitário, dividido pela população de crianças de 12-59 meses do município. No segundo estágio, a probabilidade foi calculada dividindo-se o número de crianças amostradas no setor pela população do setor censitário. Foi também levado, em conta, o fato de ter sido incluída mais de uma criança por domicílio. No comando svy$s e t$, foram declarados os fatores utilizados para o cálculo das probabilidades de seleção por meio das opções $f p c$. Foram também informados que o cluster, no primeiro estágio, foi o setor censitário, e que, no segundo estágio, o cluster foi a domicílio.

As análises bivariadas incluíram estimativas de coberturas e intervalos de $95 \%$ de confiança (IC95\%) do esquema vacinal básico incompleto segundo as variáveis independentes. O modelo final foi realizado por meio de regressão de Poisson com ajuste robusto da variância, e, para en- 
trada no modelo ajustado, foram consideradas as variáveis independentes que apresentaram valor de p inferior a 0,20 na análise bivariada, tendo permanecido no modelo final as variáveis que apresentavam $\mathrm{p}<0,10$, utilizando-se o método stepwise com eliminação retrógrada de variáveis (backward elimination).

Em respeito ao que dispõe a Resolução no 196/96 do Conselho Nacional de Saúde, esta pesquisa foi aprovada pelo Comitê de Ética em Pesquisa do Hospital Universitário Presidente Dutra (parecer no 275/06).

\section{Resultados}

Das 540 crianças estudadas, 427 (79\%) possuíam cartão de vacinas no momento da entrevista e foram avaliadas. Portanto, as perdas corresponderam a $21 \%$, sendo maiores em crianças do sexo masculino $(\mathrm{p}=0,044)$, com idade de $3-4$ anos $(\mathrm{p}=$ $0,008)$, de cor da pele preta $(\mathrm{p}=0,009)$, cujos chefes de família eram do sexo feminino $(p=0,002)$, com idade de 40-49 anos ( $\mathrm{p}=0,027)$ e sem companheiro ( $p=0,014)$ (Tabela 1$)$.

A cobertura vacinal confirmada pelo cartão de vacinas e o percentual de doses inválidas e atrasadas em crianças de 12-59 meses estão apresentados na Tabela 2 . As coberturas referentes ao esquema básico completo aos 12 meses foram maiores para doses aplicadas $(71,9 \%) \mathrm{e}$, menores para doses corretas $(23,6 \%)$. As coberturas, segundo tipo de vacina, foram mais elevadas para a vacina BCG: 99,3\% para doses aplicadas e 95,6\% para corretas. As coberturas das terceiras doses das demais vacinas foram estimadas em $81 \%$ a $85 \%$ para doses aplicadas e válidas, reduzindo-se em mais de $40 \%$ quando se consideraram doses corretas. Os maiores percentuais de doses inválidas foram para o esquema básico completo (10\%) e a segunda dose de hepatite B $(5,2 \%)$. O atraso vacinal foi mais frequente para a terceira dose da hepatite B (40\%).

As análises não ajustadas da associação entre as variáveis independentes e o esquema vacinal básico incompleto são apresentadas nas Tabelas 3 e 4. Crianças com maior percentual de esquema básico incompleto por doses aplicadas não possuíam seguro-saúde ( $\mathrm{p}=0,042$ ), eram de famílias cujos chefes tinham quatro anos ou menos de escolaridade $(\mathrm{p}=0,045)$ e pertenciam às classes econômicas D e E $(\mathrm{p}<0,001)$. Naquelas com esquema básico incompleto por doses válidas, também prevaleceram as de chefes de família das classes D e E ( $p=0,041)$. Entretanto, maior percentual de crianças que não completaram o esquema básico por doses corretas era do sexo feminino $(p=0,042)$, possuía chefes de família de cor da pele preta $(\mathrm{p}<0,001)$ e pertencia à classe econômica $\mathrm{D}(\mathrm{p}<0,001)$.

Na análise ajustada, são observadas maiores proporções de esquema básico incompleto por doses aplicadas em crianças das classes econômicas C (RP = 2,02; IC95\%: 1,01-4,02), D (RP = 3,02; IC95\%: 1,52-6,02) e E $(\mathrm{RP}=4,36$; IC95\%: $1,93-9,84)$, enquanto que os percentuais de não completude do esquema básico por doses válidas foram maiores apenas para aquelas das classes D (RP = 1,70; IC95\%: 1,14-2,52) e E (RP = 2,03; IC95\%: 1,17-3,50). Já nas crianças com esquema básico incompleto por doses corretas prevaleceram as de chefes de família das classes econômicas menos favorecidas D (RP = 1,29; IC95\%: 1,071,55) e $\mathrm{E}(\mathrm{RP}=1,30$; IC95\%: 1,01-1,66), do sexo feminino ( $R P=1,13$; IC95\%: 1,02-1,27) e cujos chefes de família eram de cor da pele preta $(\mathrm{RP}=$ 1,34; IC95\%: 1,16-1,55) (Tabela 5).

\section{Discussão}

O presente estudo evidenciou baixas coberturas das vacinas de múltiplas doses e do esquema vacinal básico completo em São Luís, quando comparado a outras pesquisas no país 16,17,18,19,20,21. As desigualdades de gênero, raciais e socioeconômicas representaram barreiras à completude do calendário básico de vacinação aos 12 meses.

A porcentagem de crianças com vacinação completa por doses aplicadas em São Luís foi inferior à encontrada em Curitiba, Brasília, Cuiabá e Teresina, em 2007, cujos valores foram superiores a $90 \%$. Percentuais inferiores aos apresentados neste estudo $(71,9 \%)$ foram encontrados em Macapá, Recife e João Pessoa, em 2007. No Brasil, a cobertura atingiu 82\% em 2007 16,20. Vale ressaltar que a comparação do esquema básico completo com critério mais rigoroso (doses corretas) não foi possível ser realizada devido à escassez de trabalhos que utilizassem esse critério, pois a maioria das pesquisas encontradas utilizou doses aplicadas.

Apenas a vacina BCG atingiu meta acima de 95\%, estabelecida pelo Ministério da Saúde, mesmo para doses corretas. Os elevados percentuais de primeira dose aplicada das vacinas tetravalente $(96,6 \%)$ e antipoliomielite $(98,6 \%)$ demonstram que as crianças estão tendo acesso aos serviços de imunização no município, centrado no conceito de acesso como entrada inicial nos serviços de saúde 22 .

Por outro lado, os baixos percentuais de cobertura para as terceiras doses, que sofreram um decréscimo acima de $40 \%$ à medida que o critério de análise se tornou mais rigoroso quando foram considerados a idade e o intervalo correto 
Tabela 1

Dados socioeconômicos e demográficos do chefe de família e das crianças de 12-59 meses segundo apresentação do cartão de vacinas. São Luís, Maranhão, Brasil, 2006

\begin{tabular}{|c|c|c|c|c|}
\hline Variáveis & $n[N=540]$ & $\begin{array}{l}\text { Amostra (com cartão) } \\
\qquad \begin{array}{c}{[n=427]} \\
n(\%)\end{array}\end{array}$ & $\begin{array}{l}\text { Perdas (sem cartão) } \\
\qquad \begin{array}{c}\text { [n = 113] } \\
n(\%)\end{array}\end{array}$ & Valor de $p$ * \\
\hline Sexo do chefe de família ** & & & & 0,002 \\
\hline Masculino & 376 & $311(82,7)$ & $65(17,3)$ & \\
\hline Feminino & 157 & $111(70,7)$ & $46(29,3)$ & \\
\hline Idade do chefe de família (anos) & & & & 0,027 \\
\hline$<30$ & 122 & $101(82,8)$ & $21(17,2)$ & \\
\hline $30-39$ & 147 & $123(83,7)$ & $24(16,3)$ & \\
\hline $40-49$ & 119 & $83(69,8)$ & $36(30,2)$ & \\
\hline$\geq 50$ & 152 & $120(78,9)$ & $32(21,1)$ & \\
\hline Escolaridade do chefe de família (anos) ** & & & & 0,498 \\
\hline$\leq 4$ & 113 & $92(81,4)$ & $21(18,6)$ & \\
\hline $5-8$ & 121 & $95(78,5)$ & $26(21,5)$ & \\
\hline $9-11$ & 226 & $174(77,0)$ & $52(23,0)$ & \\
\hline$\geq 12$ & 72 & $61(84,7)$ & $11(15,3)$ & \\
\hline Renda familiar (salários mínimos) & & & & 0,133 \\
\hline$<1$ & 121 & $94(77,7)$ & $27(22,3)$ & \\
\hline$\geq 1>3$ & 230 & $191(83,1)$ & $39(16,9)$ & \\
\hline $3>5$ & 75 & $53(70,7)$ & $22(29,3)$ & \\
\hline$>5$ & 114 & $89(78,1)$ & $25(21,9)$ & \\
\hline 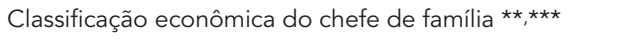 & & & & 0,846 \\
\hline A ou $B$ & 83 & $63(75,9)$ & $20(24,1)$ & \\
\hline $\mathrm{C}$ & 209 & $167(79,9)$ & $42(20,1)$ & \\
\hline D & 198 & $159(80,3)$ & $39(19,7)$ & \\
\hline $\mathrm{E}$ & 45 & $35(77,8)$ & $10(22,2)$ & \\
\hline Seguro-saúde do chefe de família & & & & 0,372 \\
\hline Sim & 127 & $104(81,9)$ & $23(18,1)$ & \\
\hline Não & 413 & $323(78,2)$ & $90(21,8)$ & \\
\hline Situação conjugal do chefe de família ** & & & & 0,014 \\
\hline Com companheiro & 390 & $319(81,8)$ & $71(18,2)$ & \\
\hline Sem companheiro & 143 & $103(72,1)$ & $40(27,9)$ & \\
\hline Inserção na população economicamente ativa do chefe & & & & 0,322 \\
\hline \multicolumn{5}{|l|}{ de família ** } \\
\hline Inserido na PEA & 497 & $394(79,3)$ & $103(20,7)$ & \\
\hline Fora da PEA & 31 & $21(67,7)$ & $10(32,3)$ & \\
\hline Cor da pele do chefe de família* & & & & 0,137 \\
\hline Branca & 140 & $104(74,3)$ & $36(25,7)$ & \\
\hline Preta & 60 & $45(75,0)$ & $15(25,0)$ & \\
\hline Parda & 329 & $269(81,8)$ & $60(18,2)$ & \\
\hline Sexo da criança & & & & 0,044 \\
\hline Masculino & 270 & $204(75,6)$ & $66(24,4)$ & \\
\hline Feminino & 270 & $223(82,6)$ & $47(17,4)$ & \\
\hline Idade da criança (anos) & & & & 0,008 \\
\hline $1-2$ & 270 & $226(83,7)$ & $44(16,3)$ & \\
\hline $3-4$ & 270 & $201(74,4)$ & $69(25,6)$ & \\
\hline
\end{tabular}

(continua) 
Tabela 1 (continuação)

\begin{tabular}{|c|c|c|c|c|}
\hline Variáveis & $n[N=540]$ & $\begin{array}{l}\text { Amostra (com cartão) } \\
\qquad \begin{array}{c}{[n=427]} \\
n(\%)\end{array}\end{array}$ & $\begin{array}{l}\text { Perdas (sem cartão) } \\
\qquad \begin{array}{c}\text { [n = 113] } \\
n(\%)\end{array}\end{array}$ & Valor de $\mathrm{p}$ * \\
\hline Cor da pele da criança * & & & & 0,009 \\
\hline Branca & 154 & $113(73,4)$ & $41(26,6)$ & \\
\hline Preta & 32 & $21(65,6)$ & $11(34,4)$ & \\
\hline Parda & 349 & $289(82,8)$ & $60(17,2)$ & \\
\hline Número de irmãos da criança & & & & 0,768 \\
\hline até 2 & 449 & $354(78,8)$ & $95(21,2)$ & \\
\hline 3 ou mais & 91 & $73(80,2)$ & $18(19,8)$ & \\
\hline Idade da mãe (anos)* & & & & 0,620 \\
\hline$<20$ & 44 & $34(77,3)$ & $10(22,7)$ & \\
\hline $20-34$ & 407 & $325(79,8)$ & $82(20,2)$ & \\
\hline$\geq 35$ & 85 & $64(75,3)$ & $21(24,7)$ & \\
\hline Recebimento de benefício monetário do governo ** & & & & 0,512 \\
\hline Sim & 107 & $87(81,3)$ & $20(18,7)$ & \\
\hline Não & 431 & $338(78,4)$ & $93(21,6)$ & \\
\hline Cadastro no Programa Saúde da Família ** & & & & 0,575 \\
\hline Sim & 180 & $145(80,6)$ & $35(19,4)$ & \\
\hline Não & 353 & $277(78,5)$ & $76(21,5)$ & \\
\hline
\end{tabular}

PEA: população economicamente ativa.

* Valor de p baseado no teste do qui-quadrado;

** Os totais diferiram devido aos valores ignorados;

*** Conforme critério da Associação Brasileira de Empresas de Pesquisas. Adoção do CCEB: Critério de Classificação Econômica Brasil.

Tabela 2

Cobertura vacinal confirmada pelo cartão de vacinas, doses inválidas e atrasadas, em crianças de 12-59 meses. São Luís, Maranhão, Brasil, 2006.

\begin{tabular}{|c|c|c|c|c|c|c|c|c|}
\hline \multirow[t]{3}{*}{ Vacinas } & \multicolumn{6}{|c|}{ Cobertura vacinal segundo doses } & \multirow{3}{*}{$\begin{array}{c}\text { Doses } \\
\text { inválidas } \\
\text { (\%) }\end{array}$} & \multirow{3}{*}{$\begin{array}{c}\text { Doses } \\
\text { atrasadas } \\
\text { (\%) }\end{array}$} \\
\hline & \multicolumn{2}{|c|}{ Aplicadas } & \multicolumn{2}{|c|}{ Válidas } & \multicolumn{2}{|c|}{ Corretas } & & \\
\hline & $n(\%)$ & IC95\% & n (\%) & IC95\% & $n(\%)$ & IC95\% & & \\
\hline BCG & $424(99,3)$ & $97,9-99,8$ & - & & $408(95,6)$ & $93,1-97,3$ & - & 3,7 \\
\hline Hepatite B (1a dose) & $423(99,1)$ & $97,6-99,7$ & - & & $375(87,8)$ & $84,4-90,8$ & - & 11,3 \\
\hline Hepatite B (2a dose) & $415(97,2)$ & $95,1-98,5$ & $393(92,0)$ & $89,1-94,4$ & $273(63,9)$ & $59,2-68,5$ & 5,2 & 28,1 \\
\hline Hepatite B (3a dose) & $367(85,9)$ & $82,3-89,1$ & $346(81,0)$ & $76,9-84,6$ & $175(40,9)$ & $36,3-45,8$ & 4,9 & 40,1 \\
\hline Antipoliomielite (1 $\underline{a}$ dose) & $421(98,6)$ & $96,9-99,5$ & $405(94,8)$ & $92,3-96,7$ & $340(79,6)$ & $75,5-83,3$ & 3,8 & 15,2 \\
\hline Antipoliomielite (2a dose) & $412(96,5)$ & $94,3-98,0$ & $396(92,7)$ & $89,8-95,0$ & $264(61,8)$ & $57,0-66,5$ & 3,8 & 30,9 \\
\hline Antipoliomielite (3a dose) & $366(85,7)$ & $82,0-88,9$ & $355(83,1)$ & $79,2-86,6$ & $211(49,4)$ & $44,6-54,3$ & 2,6 & 33,7 \\
\hline DTP ou tetravalente (1 $\underline{a}$ dose) & $411(96,3)$ & $93,9-97,8$ & $408(95,5)$ & $93,1-97,3$ & $334(78,2)$ & $73,4-82,0$ & 0,8 & 17,3 \\
\hline DTP ou tetravalente (3a dose) & $350(81,9)$ & $77,9-85,4$ & $346(81,0)$ & $76,9-84,6$ & $209(48,9)$ & $44,1-53,8$ & 0,9 & 32,1 \\
\hline Esquema básico completo * & $307(71,9)$ & $67,4-76,1$ & $264(61,8)$ & $57,0-66,4$ & $101(23,6)$ & $19,7-27,9$ & 10,1 & 38,2 \\
\hline
\end{tabular}

IC95\%: intervalo de 95\% de confiança.

* Foi considerado esquema básico completo aos 12 meses: 1 dose de BCG, 3 de antipoliomielite, 3 de hepatite e 3 de DTP ou tetravalente. 
Análise não ajustada da associação entre fatores socioeconômicos e demográficos em crianças de 12-59 meses e esquema vacinal básico incompleto. São Luís, Maranhão, Brasil, 2006.

\begin{tabular}{|c|c|c|c|c|c|c|c|c|c|}
\hline \multirow[t]{3}{*}{ Variáveis } & \multicolumn{9}{|c|}{ Esquema vacinal básico incompleto segundo doses } \\
\hline & \multicolumn{3}{|c|}{ Aplicadas } & \multicolumn{3}{|c|}{ Válidas } & \multicolumn{3}{|c|}{ Corretas } \\
\hline & n (\%) & RP (IC95\%) & $\begin{array}{l}\text { Valor } \\
\text { de } p\end{array}$ & n (\%) & RP (IC95\%) & $\begin{array}{l}\text { Valor } \\
\text { de } p\end{array}$ & n (\%) & RP (IC95\%) & $\begin{array}{l}\text { Valor } \\
\text { de } p\end{array}$ \\
\hline Sexo da criança & & & 0,726 & & & 0,591 & & & 0,042 \\
\hline Masculino & $59(28,9)$ & 1,00 & & $75(36,8)$ & 1,00 & & $146(71,6)$ & 1,00 & \\
\hline Feminino & $61(27,4)$ & $0,94(0,69-1,29)$ & & $88(39,5)$ & $1,07(0,82-1,39)$ & & $180(80,7)$ & $1,13(1,01-1,27)$ & \\
\hline Idade da criança (anos) & & & 0,465 & & & 0,811 & & & 0,697 \\
\hline $1-2$ & $60(26,6)$ & $0,89(0,64-1,22)$ & & $85(37,6)$ & $0,97(0,53-1,26)$ & & $171(75,7)$ & $0,98(0,89-1,08)$ & \\
\hline $3-4$ & $60(29,9)$ & 1,00 & & $78(38,8)$ & 1,00 & & $155(77,1)$ & 1,00 & \\
\hline Cor da pele da criança * & & & 0,559 & & & 0,513 & & & 0,249 \\
\hline Branca & $29(25,7)$ & $0,91(0,62-1,35)$ & & $38(33,6)$ & $0,85(0,64-1,13)$ & & $78(69,0)$ & $0,88(0,75-1,02)$ & \\
\hline Preta & $8(38,1)$ & $1,36(0,69-2,68)$ & & $9(42,8)$ & $1,08(0,61-1,92)$ & & $17(80,9)$ & $1,03(0,83-1,27)$ & \\
\hline Parda & $81(28,0)$ & 1,00 & & $114(39,4)$ & 1,00 & & $227(78,5)$ & 1,00 & \\
\hline Número de irmãos da criança & & & 0,698 & & & 0,489 & & & 0,764 \\
\hline Até 2 & $98(27,7)$ & 1,00 & & $138(38,9)$ & 1,00 & & $269(75,9)$ & 1,00 & \\
\hline 3 ou + & $22(30,1)$ & $1,08(0,70-1,68)$ & & $25(34,3)$ & $0,88(0,60-1,28)$ & & $57(78,0)$ & $1,02(0,86-1,23)$ & \\
\hline Idade da mãe * & & & 0,693 & & & 0,783 & & & 0,135 \\
\hline$<20$ & $10(29,4)$ & $1,08(0,57-2,05)$ & & $14(41,2)$ & $1,10(0,69-1,28)$ & & $27(79,5)$ & $1,06(0,87-1,30)$ & \\
\hline $20-34$ & $88(27,1)$ & 1,00 & & $121(37,2)$ & 1,00 & & $242(74,5)$ & 1,00 & \\
\hline$\geq 35$ & $21(32,8)$ & $1,21(0,77-1,89)$ & & $27(43,2)$ & $1,13(0,78-1,63)$ & & $54(84,4)$ & $1,13(1,00-1,28)$ & \\
\hline Benefício monetário do governo ** & & & 0,569 & & & 0,570 & & & 0,600 \\
\hline Sim & $93(27,5)$ & 1,00 & & $36(41,4)$ & 1,00 & & $64(73,6)$ & 1,00 & \\
\hline Não & $27(31,0)$ & $0,88(0,58-1,35)$ & & $127(37,6)$ & $0,91(0,65-1,27)$ & & $260(76,9)$ & $1,04(0,88-1,24)$ & \\
\hline Programa Saúde da Família * & & & 0,471 & & & 0,723 & & & 0,562 \\
\hline Sim & $44(30,3)$ & 1,00 & & $57(39,3)$ & 1,00 & & $113(77,9)$ & 1,00 & \\
\hline Não & $74(26,7)$ & $0,88(0,62-1,25)$ & & $104(37,5)$ & $0,95(0,74-1,24)$ & & $208(75,1)$ & $0,96(0,85-1,09)$ & \\
\hline Seguro-saúde & & & 0,042 & & & 0,268 & & & 0,157 \\
\hline $\operatorname{Sim}$ & $20(19,2)$ & 1,00 & & $34(32,7)$ & 1,00 & & $74(71,1)$ & 1,00 & \\
\hline Não & $100(30,9)$ & $1,60(1,01-2,55)$ & & $129(39,9)$ & $1,22(0,85-1,75)$ & & $252(78,0)$ & $1,09(0,96-1,25)$ & \\
\hline
\end{tabular}

* Os totais diferiram devido aos valores ignorados.

preconizados, levaram a uma drástica diminuição na cobertura do esquema básico completo (de $71,9 \%$ para $23,6 \%$ ) e apontam falhas no programa de imunização. Apesar de $71,9 \%$ das crianças estarem completamente vacinadas aos 12 meses por doses aplicadas, 76,4\% não estão vacinadas adequadamente.

$\mathrm{O}$ atraso do esquema básico foi constatado em $38,2 \%$ das crianças, superior ao percentual encontrado em Cachoeirinha (Rio Grande do Sul) 23. O atraso em todas as vacinas de múltiplas doses foi responsável por essa elevada proporção, assim como em outros estudos 16,23. Estratégias de busca ativa dos faltosos precisam ser implementadas. Acredita-se que situações ligadas às ações dos profissionais, como receio de abrir frascos multidoses para apenas uma ou duas crianças para não aumentar a perda de doses de vacina, tenham contribuído para a não vacinação oportuna 24 . Além disso, a superestimação das contraindicações possibilita o adiamento desnecessário de doses e pode levar à perda de imunizar a criança definitivamente 25 . Mesmo que a administração de imunobiológicos em intervalos superiores ao preconizado não reduza a concentração de anticorpos, a ausência de administração da última dose não estabelece imunidade, deixando o indivíduo propenso, mesmo que parcialmente, às doenças preveníveis por vacinação ${ }^{10}$. Além disso, o acúmulo de indivíduos suscetíveis oferece risco à proteção individual e coletiva, facilitando a 
Análise não ajustada da associação entre fatores socioeconômicos e demográficos do chefe de família de crianças de 12-59 meses e esquema vacinal básico incompleto. São Luís, Maranhão, Brasil, 2006.

\begin{tabular}{|c|c|c|c|c|c|c|c|c|c|}
\hline \multirow[t]{3}{*}{ Variáveis } & \multicolumn{9}{|c|}{ Esquema vacinal básico incompleto segundo doses } \\
\hline & \multicolumn{3}{|c|}{ Aplicadas } & \multicolumn{3}{|c|}{ Válidas } & \multicolumn{3}{|c|}{ Corretas } \\
\hline & n (\%) & RP (IC95\%) & $\begin{array}{l}\text { Valor } \\
\text { de } p\end{array}$ & $n(\%)$ & RP (IC95\%) & $\begin{array}{l}\text { Valor } \\
\text { de } p\end{array}$ & n (\%) & RP (IC95\%) & $\begin{array}{l}\text { Valor } \\
\text { de } p\end{array}$ \\
\hline Sexo* & & & 0,872 & & & 0,699 & & & 0,911 \\
\hline Masculino & $87(27,9)$ & $0,97(0,67-1,40)$ & & $117(37,6)$ & $0,94(0,72-1,24)$ & & $237(76,2)$ & $1,00(0,88-1,14)$ & \\
\hline Feminino & $32(28,8)$ & 1,00 & & $44(39,6)$ & 1,00 & & $84(75,6)$ & 1,00 & \\
\hline Idade (anos) & & & 0,547 & & & 0,478 & & & 0,831 \\
\hline$<30$ & $31(30,7)$ & $1,27(0,84-1,91)$ & & $44(43,6)$ & $1,24(0,85-1,81)$ & & $80(79,2)$ & $1,08(0,92-1,27)$ & \\
\hline $30-39$ & $33(26,8)$ & $1,11(0,70-1,75)$ & & $43(34,9)$ & $0,99(0,68-1,47)$ & & $94(76,4)$ & $1,04(0,89-1,22)$ & \\
\hline $40-49$ & $27(32,5)$ & $1,34(0,87-2,07)$ & & $34(40,9)$ & $1,17(0,79-1,73)$ & & $64(77,1)$ & $1,05(0,89-1,24)$ & \\
\hline$\geq 50$ & $29(24,2)$ & 1,00 & & $42(35,0)$ & 1,00 & & $88(73,3)$ & 1,00 & \\
\hline Escolaridade (anos) * & & & 0,045 & & & 0,170 & & & 0,221 \\
\hline$\leq 4$ & $35(38,0)$ & $0,01(1,11-2,22)$ & & $44(47,8)$ & $1,38(1,00-1,91)$ & & $78(84,8)$ & $1,16(1,00-1,34)$ & \\
\hline $5-8$ & $26(27,4)$ & $0,61(0,69-1,87)$ & & $36(37,9)$ & $1,09(0,78-1,53)$ & & $70(73,7)$ & $1,01(0,86-1,19)$ & \\
\hline $9-11$ & $42(24,1)$ & 1,00 & & $60(34,5)$ & 1,00 & & $127(72,9)$ & 1,00 & \\
\hline$\geq 12$ & $16(26,2)$ & $0,77(0,61-1,93)$ & & $21(34,4)$ & $0,99(0,64-1,55)$ & & $46(75,4)$ & $1,03(0,87-1,22)$ & \\
\hline Renda familiar (salários mínimos) & & & 0,345 & & & 0,294 & & & 0,442 \\
\hline Até 1 & $34(36,2)$ & $1,60(0,94-2,74)$ & & $40(42,6)$ & $1,30(0,84-2,02)$ & & $78(82,9)$ & $1,10(0,93-1,31)$ & \\
\hline$\geq 1-3$ & $50(26,2)$ & $1,16(0,72-1,89)$ & & $70(36,7)$ & $1,12(0,78-1,63)$ & & $141(73,8)$ & $0,98(0,84-1,14)$ & \\
\hline$>3-5$ & $16(30,2)$ & $1,34(0,74-2,41)$ & & $24(45,3)$ & $1,39(0,94-2,04)$ & & $40(75,5)$ & $1,00(0,79-1,26)$ & \\
\hline$>5$ & $20(22,5)$ & 1,00 & & $29(32,6)$ & 1,00 & & $67(75,3)$ & 1,00 & \\
\hline Classificação econômica *,** & & & $<0,001$ & & & 0,041 & & & 0,005 \\
\hline$A$ ou $B$ & $10(15,8)$ & 1,00 & & $16(25,4)$ & 1,00 & & $41(65,1)$ & 1,00 & \\
\hline C & $39(23,3)$ & $1,47(0,79-2,73)$ & & $62(37,1)$ & $1,46(0,90-2,37)$ & & $118(70,7)$ & $1,08(0,88-1,34)$ & \\
\hline $\mathrm{D}$ & $53(33,3)$ & $2,10(1,23-3,57)$ & & $66(41,5)$ & $1,63(1,08-2,47)$ & & $134(84,3)$ & $1,29(1,06-1,59)$ & \\
\hline E & $17(48,5)$ & $3,06(1,61-5,82)$ & & $18(51,4)$ & $2,02(1,16-3,52)$ & & $30(85,7)$ & $1,32(0,97-1,74)$ & \\
\hline Situação conjugal & & & 0,410 & & & 0,942 & & & 0,665 \\
\hline Com companheiro & $93(29,2)$ & 1,00 & & $122(38,2)$ & 1,00 & & $241(75,5)$ & 1,00 & \\
\hline Sem companheiro & $26(25,2)$ & $0,86(0,61-1,22)$ & & $39(37,9)$ & $0,99(0,75-1,30)$ & & $80(77,7)$ & $1,03(0,90-1,17)$ & \\
\hline Inserção na PEA * & & & 0,164 & & & 0,182 & & & 0,267 \\
\hline Inserido na PEA & $107(27,2)$ & 1,00 & & $147(37,3)$ & 1,00 & & $299(75,9)$ & 1,00 & \\
\hline Fora da PEA & $8(40,0)$ & $1,47(0,85-2,55)$ & & $10(50,0)$ & $1,34(0,86-2,07)$ & & $17(85,0)$ & $1,12(0,91-1,37)$ & \\
\hline Cor da pele * & & & 0,792 & & & 0,625 & & & $<0,001$ \\
\hline Branca & $31(29,8)$ & 1,00 & & $38(36,5)$ & 1,00 & & $71(68,3)$ & 1,00 & \\
\hline Preta & $14(31,1)$ & $1,04(0,59-1,86)$ & & $20(44,4)$ & $1,21(0,79-1,87)$ & & $42(93,3)$ & $1,37(1,19-1,57)$ & \\
\hline Parda & $72(26,8)$ & $0,89(0,58-1,39)$ & & $101(37,5)$ & $1,03(0,72-1,46)$ & & $205(76,2)$ & $1,17(0,97-1,29)$ & \\
\hline
\end{tabular}

IC95\%: intervalo de 95\% de confiança; PEA: população economicamente ativa.

* Os totais diferiram devido aos valores ignorados;

** Conforme critério da Associação Brasileira de Empresas de Pesquisas. Adoção do CCEB: Critério de Classificação Econômica Brasil.

introdução e manutenção de agentes infecciosos 9,10. Nos Estados Unidos, os bolsões desses suscetíveis têm resultado em surtos de doenças imunopreveníveis 26 .

Outro problema encontrado foi a elevada proporção de doses inválidas, em comparação com o estudo de Luhm et al. 16. Essa taxa foi mais evidente nas vacinas contra Hepatite B e Tetravalente, demonstrando, possivelmente, falha no agendamento ou na indicação correta dessas vacinas. Estudo realizado por Paulo 24, na região norte do Município de São Paulo, revelou elevada 
Análise ajustada da associação entre características socioeconômicas e demográficas do chefe de família e das crianças de 12-59 meses e esquema vacinal básico incompleto. São Luís, Maranhão, Brasil, 2006.

\begin{tabular}{|c|c|c|c|c|c|c|}
\hline \multirow[t]{3}{*}{ Variáveis } & \multicolumn{6}{|c|}{ Esquema vacinal básico incompleto segundo doses } \\
\hline & \multicolumn{2}{|c|}{ Aplicadas *,** } & \multicolumn{2}{|c|}{ Válidas *,** } & \multicolumn{2}{|c|}{ Corretas $*, \star *$} \\
\hline & RP (IC95\%) & Valor de $p$ & RP (IC95\%) & Valor de $p$ & RP (IC95\%) & Valor de $p$ \\
\hline Classificação econômica do chefe de família & & 0,003 & & 0,029 & & 0,006 \\
\hline A ou B & 1,00 & & 1,00 & & 1,00 & \\
\hline C & $2,02(1,01-4,02)$ & & $1,51(0,94-2,42)$ & & $1,08(0,89-1,32)$ & \\
\hline $\mathrm{D}$ & $3,02(1,52-6,02)$ & & $1,70(1,14-2,52)$ & & $1,29(1,07-1,55)$ & \\
\hline $\mathrm{E}$ & $4,36(1,93-9,84)$ & & $2,03(1,17-3,50)$ & & $1,30(1,01-1,66)$ & \\
\hline Cor da pele do chefe de família & & & & & & $<0,001$ \\
\hline Branca & & & & & 1,00 & \\
\hline Preta & & & & & $1,34(1,16-1,55)$ & \\
\hline Parda & & & & & $1,05(0,92-1,21)$ & \\
\hline Sexo da criança & & & & & & 0,025 \\
\hline Masculino & & & & & 1,00 & \\
\hline Feminino & & & & & $1,13(1,02-1,27)$ & \\
\hline
\end{tabular}

IC95\%: intervalo de 95\% de confiança.

* Ajustadas pelo modelo de regressão de Poisson (foram incluídas apenas as variáveis com $p<0,20$ na análise não ajustada);

** $n=417$, no modelo para doses aplicadas; $n=414$, no modelo para doses válidas; e $n=418$, no modelo para doses corretas.

proporção de profissionais que não consideraram o intervalo mínimo entre as doses e administraram vacinas antes da data recomendada. A não administração de imunobiológicos na idade oportuna, seguindo os intervalos preconizados, pode reduzir a resposta imunológica, diminuindo a eficácia das vacinas 10. Uma possível explicação para isso seria que os profissionais de saúde estejam encurtando os intervalos entre as doses das vacinas como forma de evitar oportunidades perdidas durante a procura pelo serviço de saúde, em qualquer ocasião ou em campanhas de vacinação. Possivelmente, mesmo sendo capacitados para tal atividade e conhecendo as normas gerais do programa, é provável que eles administrem doses de vacina poucos dias antes do agendamento, achando que isso não faça diferença para a imunização. Talvez o desconhecimento da importância de manter os intervalos preconizados como indispensável fator para a adequação imunológica seja uma razão para essa prática. É necessário incrementar a supervisão dos enfermeiros nas salas de vacinas, de modo contínuo e regular, identificando e intervindo oportunamente e garantindo o cumprimento das normas vigentes.

Apesar dessas lacunas, destaca-se que, de 1994 a 2006, houve um incremento de 60,3\% na cobertura do esquema vacinal completo (de $44,1 \%$ para $71,9 \%$ ) e de $40 \%$ nas coberturas das vacinas isoladamente: BCG (de $72,4 \%$ para $99,3 \%$ ), DTP (de $57 \%$ para $81,9 \%$ ), Pólio (de $59,9 \%$ para $85,7 \%$ ), quando foram comparados esses dados com inquérito anterior realizado, em São Luís, por Silva et al. ?.

Ainda que não se possa afirmar, é possível supor que o PSF e o recebimento de benefício de transferência de renda do governo, a exemplo do Bolsa Família, possam ter contribuído, em parte, para elevar os percentuais de cobertura vacinal no município, pois as coberturas foram iguais quando foram comparados participantes com não participantes desses programas. Barata et al. 20 sugerem que o programa de imunização do Brasil tem sido bem-sucedido em alcançar alta cobertura vacinal entre as crianças menos favorecidas. Como esses programas atendem em maior proporção populações menos favorecidas, seriam esperados menores percentuais de vacinação nesses grupos se essas estratégias não tivessem efeito na vacinação. Porém, as coberturas nesses grupos foram semelhantes, indicando, possivelmente, um caminho para elevar as coberturas vacinais dos menos favorecidos que não são atendidos por esses programas.

Esse provável achado poderia ser explicado, em parte, pelas ações do PSF no município. Mesmo sendo baixa a cobertura desse programa (30\%), houve uma ampliação do número de equipes, passando de uma equipe, implantada 
em 1994, para 82 em 2004 27,28. Alguns estudos têm demonstrado melhores coberturas vacinais em crianças atendidas por essas equipes, salientando a importância do trabalho dos agentes comunitários de saúde na verificação das carteiras de vacina e na busca das crianças faltosas durante as visitas domiciliares 16,29,30,31,32. Além disso, o recebimento de benefícios sociais do governo está vinculado à obrigatoriedade de se manter o cartão de vacinas da criança em dia. Possivelmente, essa estratégia esteja alcançando também uma parcela das crianças menos favorecidas na medida em que os pais se preocupam mais com essa questão 33 .

Embora trabalhos recentes mostrem coberturas mais elevadas em segmentos mais pobres da população $16,17,20$, neste estudo, crianças das classes econômicas menos favorecidas apresentaram maior risco de não completarem as vacinas, apontando uma desigualdade social na cobertura vacinal. Menores percentuais de cobertura nos mais pobres foram observados também em Portugal 19 e em Cachoeirinha 23.

Não foram observadas diferenças na cobertura vacinal segundo a renda familiar, assim como há 12 anos 7 . A classificação econômica da ABEP, que leva, em consideração, a posse de bens e a escolaridade do chefe, provavelmente se mostrou um indicador mais sensível para mensurar essas desigualdades do que a renda. É possível que maiores erros de mensuração sejam encontrados na medida da renda, pois algumas pessoas não a informam com exatidão. Esses resultados são preocupantes, pois, segundo Tertuliano \& Stein 23 , nas famílias com menores condições socioeconômicas, as atividades preventivas não são consideradas prioridade.

Dessa forma, os resultados sugerem que as disparidades socioeconômicas na vacinação encontradas no estudo de Silva et al. 7, em 1994, possivelmente se mantiveram ao longo dos doze anos, pois os indicadores socioeconômicos continuaram influenciando o não cumprimento do calendário vacinal básico, sendo ressalvadas as diferenças nas variáveis de mensuração dessa medida.

Em 1994, a menor escolaridade materna mostrou-se uma barreira socioeconômica à não completude do esquema básico de vacinação 7 . Em 2006, a escolaridade investigada foi a do chefe de família, não sendo observada diferença de cobertura segundo essa variável. Outros estudos também demonstraram não haver associação entre a escolaridade dos responsáveis e a condição vacinal 25,34,35. O inquérito nacional realizado por Barata et al. 20, utilizando escolaridade materna, não revelou associação do grau de instrução com a vacinação incompleta. Talvez o ní- vel de escolaridade não esteja mais interferindo na cobertura vacinal em São Luís, assim como em outros locais do país 7,20,34,35, possivelmente por maior conscientização dos pais.

Crianças de chefes de família de cor da pele preta apresentaram menores percentuais de vacinação, o que não se explicou pela condição socioeconômica. As diferenças nos percentuais de cobertura segundo a cor da pele se mantiveram mesmo após ajuste para fatores socioeconômicos, sugerindo que essas crianças tenham maiores dificuldades de serem vacinadas, independentemente da classe econômica. No entanto, em estudo realizado em capitais brasileiras, a raça não foi associada com a vacinação incompleta 20. Uma provável explicação seria a discriminação racial no acesso aos serviços de vacinação para crianças de pais negros. Segundo o UNICEF 1, crianças podem ser excluídas dos serviços de saúde devido a atitudes negativas de discriminação dos pais, o que reduz acentuadamente a possibilidade de serem alcançadas por intervenções que podem salvar vidas. É possível que o mito de "democracia racial" no Brasil ainda seja uma barreira para a detecção dessas diferenças, pois poucas pessoas admitem o seu preconceito em relação à cor da pele 36,37 .

Maiores percentuais de esquema básico incompleto foram encontrados em crianças do sexo feminino. Alguns autores consideram que há uma proteção de crianças do sexo masculino pelas famílias 38,39. Ao contrário dos nossos achados, estudos recentes não evidenciaram diferença de gênero na cobertura vacinal 16,20,23. Diferenças na cobertura vacinal de acordo com o sexo provavelmente sejam explicadas por diferentes padrões socioculturais e de compreensão do papel de cada sexo na sociedade 40 .

$\mathrm{Na}$ análise ajustada, foram observadas desigualdades socioeconômicas na vacinação, mensuradas pela Classificação Econômica Brasil pelos três critérios utilizados (segundo doses aplicadas, válidas e corretas), sendo que o risco de não completar o esquema básico de vacinação aumentou para as classes D e E à medida em que o critério foi mais rigoroso. Além do mais, pelo critério mais rigoroso, segundo doses corretas, surgiram também desigualdades raciais e de gênero na cobertura vacinal, o que pode ser atribuído ao atraso vacinal que foi mais comum nas crianças cujos chefes de família relataram cor da pele preta.

Destacam-se como pontos fortes desta pesquisa: (1) ser um estudo de base populacional, sendo que a maioria dos estudos comparados utilizou dados secundários de sistemas informatizados ou de unidades de saúde; (2) ser um estudo que avaliou a cobertura vacinal por doses 
aplicadas, válidas e corretas, quando a maioria dos estudos encontrados utilizaram apenas doses aplicadas para esquema básico completo.

De outro modo, teve, como limitação, o fato de o estudo ter utilizado dados de um inquérito de saúde mais amplo que não se voltou exclusivamente para avaliar a cobertura vacinal em menores de cinco anos 12, portanto, informações relacionadas à utilização dos serviços de vacinação não puderam ser investigadas.

O número de perdas ligeiramente superior a $20 \%$ pela não apresentação do cartão de vacinas também foi uma limitação, pois elas não foram homogêneas. É possível que as estimativas de cobertura estejam levemente subestimadas, pois houve maior participação na amostra de crianças do sexo feminino, o que pode ter interferido nos resultados para esse grupo.

Neste estudo, não se utilizou regressão logística, empregada em estudos transversais com desfechos binários, pois estima a razão de chances e não de prevalências. Em pesquisas como esta, em que a prevalência é elevada, a razão de chances pode superestimar a razão de prevalências, podendo distorcer a interpretação 41 . O trabalho de Tertuliano \& Stein 23 , que avaliou atraso vacinal e seus determinantes, mostrou uma superestimação das prevalências com a utilização da regressão logística, reconhecida por seus autores. Dessa forma, para minimizar possíveis erros de estimação, optou-se pela regressão de Poisson.

Por fim, entende-se que o melhor caminho para reduzir o risco da vulnerabilidade dessas crianças é o alcance de altos índices de vacinação e o cumprimento do calendário básico adequadamente, no menor período de tempo possível, garantindo a eficácia das vacinas. O principal desafio agora reside na garantia dos serviços de vacinação e na educação dos profissionais e responsáveis para o alcance das crianças mais afetadas, além do monitoramento dessas estratégias. Talvez a implantação de um registro informatizado de imunização seja um caminho de apoio ao cumprimento do esquema básico de vacinação, minimizando as falhas de agendamento, como se tem observado em Curitiba 16.

Os dados demonstram que há necessidade de reforçar as políticas equitativas que eliminem as desigualdades sociais ainda presentes nas coberturas vacinais em São Luís para que essas atinjam níveis capazes de assegurar proteção contra doenças imunopreveníveis a todas as crianças. Políticas afirmativas poderiam minimizar a iniquidade de acesso e facilitar a inserção de crianças de famílias negras nos serviços de vacinação de maneira mais justa e igualitária, aumentando as coberturas vacinais em São Luís.

\section{Resumen}

El estudio tiene como objetivo evaluar la cobertura de vacunación y los factores asociados al esquema incompleto de vacunación básico a los 12 meses de edad, realizado en 427 niños de 12 a 59 meses, en São Luis, Maranhão, Brasil, 2006. Se trata de una encuesta domiciliaria transversal, de base poblacional, con muestreo complejo por conglomerados. Se empleó la regresión de Poisson con un ajuste robusto de variancia. La cobertura para el esquema básico completo fue de un 71,9\% según las dosis aplicadas, un 61,8\% para dosis válidas y un 23,6\% para dosis correctas. Las vacunas contra la hepatitis B y tetravalente presentaron mayores porcentajes de dosis aplicadas en fechas o intervalos inferiores a los recomendados. Los porcentajes de atraso en la vacunación fueron elevados, excepto para la vacuna BCG. Los porcentajes más elevados de esquema incompleto de vacunación básico se encontraron en niños de clases económicas $D$ y $E$, de sexo femenino y con jefes de familia afrobrasileños. Las desigualdades raciales, de género y socioeconómicas representaron barreras a la vacunación completa, indicando la necesidad de reforzar las políticas equitativas que eliminen esas desigualdades.

Inmunización; Cobertura de Vacunación; Salud de Niño 


\section{Colaboradores}

A. V. C. P. Yokokura concebeu o estudo, revisou a literatura, realizou as análises e interpretação dos dados, redigiu o manuscrito e contribuiu com a redação. A. A. M. Silva coordenou o projeto, realizou as análises e interpretação dos dados e contribuiu com a redação. A. C. F. Bernardes revisou a literatura, interpretou as análises e contribuiu com a redação. F. Lamy Filho participou da concepção geral do projeto, interpretação dos resultados e redação. M. T. S. S. B. Alves participou da concepção geral do projeto, interpretação dos resultados e redação. N. A. L. Cabra revisou a literatura, realizou as análises, interpretou os dados e contribuiu com a redação. R. F. L. B. Alves participou da concepção geral do projeto, interpretação dos resultados e redação.

\section{Referências}

1. Fundo das Nações Unidas para a Infância. Situação mundial da infância 2008: sobrevivência infantil. Brasília: Fundo das Nações Unidas para a Infância; 2007.

2. Organización Mundial de la Salud; Fondo de las Naciones Unidas para la Infancia; Banco Mundial. Vacunas e inmunización: situación mundial. Geneva: Organización Mundial de la Salud; 2010.

3. World Health Organization. Meeting of the immunization Strategic Advisory Group of Experts, November 2008 - conclusions and recommendations. Wkly Epidemiol Rec 2009; 84:1-16.

4. Secretaria de Vigilância em Saúde, Ministério da Saúde. Programa Nacional de Imunização - 30 anos. Brasília: Ministério da Saúde; 2003.

5. Pereira MAD, Barbosa SRS. O cuidado de enfermagem na imunização: os mitos e as verdades. Revista Meio Ambiente e Saúde 2007; 2:76-88.

6. Mota E. Inquérito domiciliar de cobertura vacinal: a perspectiva do estudo das desigualdades sociais no acesso à imunização básica infantil. Rev Bras Epidemiol 2008; 11 Suppl 1:S125-8.

7. Silva AAM, Gomes UA, Tonial SR, Silva RA. Cobertura vacinal e fatores de risco associados à nãovacinação em localidade urbana do Nordeste brasileiro. Rev Saúde Pública 1999; 33:147-56.
8. Molina AC, Godoy I, Carvalho LR, Caldas JAL. Situação vacinal infantil e características individuais e familiares do interior de São Paulo. Rev Acta Sci Healh Sci 2007; 29:99-106.

9. Moraes JC. Inquérito de cobertura vacinal nas áreas urbanas das capitais do Brasil - cobertura vacinal 2007. São Paulo: Centro de Estudos Augusto Leopoldo Ayrosa Galvão; 2007.

10. Coordenação Geral do Programa Nacional de Imunizações, Centro Nacional de Epidemiologia, Fundação Nacional de Saúde. Manual de normas de vacinação. Brasília: Ministério da Saúde; 2001.

11. Luhm KR. Cobertura vacinal e fatores associados à situação vacinal em Curitiba [Tese de Doutorado]. São Paulo: Faculdade de Saúde Pública, Universidade de São Paulo; 2008.

12. Alves MTSB, Sousa SMPS, Coimbra LC. Atenção à saúde do adulto e da criança no Maranhão. São Luís: EDUFMA; 2009.

13. Ministério da Saúde. Portaria GM no 1.602, de 17 de julho de 2006. Institui em todo o território nacional os calendários de vacinação. Diário Oficial da União 2006; 18 jul. 
14. Coordenação Geral do Programa Nacional de Imunizações, Centro Nacional de Epidemiologia, Fundação Nacional de Saúde. Manual de procedimentos para vacinação. Brasília: Ministério da Saúde; 2001.

15. Lee ES, Forthofer RN, Lorimer RJ. Analyzing complex survey data. $2^{\text {th }}$ Ed. Newbury Park: Sage; 2006.

16. Luhm KR, Cardoso MRA, Waldman EA. Cobertura vacinal em menores de dois anos a partir de registro informatizado de imunização em Curitiba, PR. Rev Saúde Pública 2011; 45:90-8.

17. Moraes JC, Ribeiro MCSA. Desigualdades sociais e cobertura vacinal: uso de inquéritos domiciliares. Rev Bras Epidemiol 2008; 11 Suppl 1:S113-24.

18. Pereira DR, Matias TAF, Soares DFPP, Carvalho WO. Cobertura Vacinal em crianças de 12 a 23 meses de idade: estudo exploratório tipo survey. Rev Eletrônica Enferm 2009; 11:360-67. http://www.fen.ufg. $\mathrm{br} / \mathrm{revista} / \mathrm{v} 11 / \mathrm{n} 2 / \mathrm{v} 11 \mathrm{n} 2 \mathrm{a} 17 . \mathrm{htm}$ (acessado em 13/Set/2010).

19. Rocha R, Sampaio MJ, Pereira CA, Liberal I. Fatores associados ao não cumprimento do Programa Nacional de Vacinação e das vacinas pneumocócica conjugada heptavalente e contra o rotavírus. Acta Pediátrica Portuguesa 2010; 415:195-200.

20. Barata RB, Ribeiro MC, de Moraes JC, Flannery B; Vaccine Coverage Survey 2007 Group. Socioeconomic inequalities and vaccination coverage: results of an immunisation coverage survey in 27 Brazilian capitals, 2007-2008. J Epidemiol Community Health 2012; 66:934-41.

21. Cesar JA, Mendoza-Sassi R, Horta BL, Ribeiro PRP, Ávila AC, Santos FM, et al. Indicadores básicos de saúde infantil em área urbana no extremo sul do Brasil: estimando prevalências e avaliando diferenciais. J Pediatr (Rio J.) 2006; 82:437-44.

22. Travassos C, Martins M. Uma revisão sobre os conceitos de acesso e utilização de serviços de saúde. Cad Saúde Pública 2004; 20 Suppl 2:S190-8.

23. Tertuliano GC, Stein AT. Atraso vacinal e seus determinantes: um estudo em localidade atendida pela Estratégia de Saúde da Família. Ciênc Saúde Coletiva 2011; 16:523-30.

24. Paulo EF. Oportunidades perdidas de vacinação de crianças menores de dois anos de idade, ocorridas nas salas de vacinação nas unidades de saúde na região do norte do município de São Paulo [Dissertação de Mestrado]. São Paulo: Programa de Pós-graduação em Ciências, Coordenadoria de Controle de Doenças, Secretaria de Estado da Saúde de São Paulo; 2010.

25. Miranda AS, Scheibel IM, Tavares MRG, Takeda SMR. Avaliação da cobertura vacinal do esquema básico para o primeiro ano de vida. Rev Saúde Pública 1995; 29:208-14.

26. Omer SB, Salmon DA, Orenstein WA, Hart P, Halsey $\mathrm{N}$. Vaccine refusal, mandatory immunization, and the risks of vaccine preventable diseases. N Engl J Med 2009; 360:1981-8.

27. Nobre RNLL. Saúde da família: uma utopia realizável [Dissertação de Mestrado]. São Luís: Universidade Federal do Maranhão; 1999.
28. Nicolau LS. A Estratégia Saúde da Família no município de São Luís: avanços e desafios [Dissertação de Mestrado]. Brasília: Universidade de Brasília; 2008.

29. Secretaria de Políticas de Saúde, Ministério da Saúde. Guia prático do Programa Saúde da Família. Brasília: Ministério da Saúde; 2001.

30. Cruz MGB. Estudo comparativo de alguns indicadores em municípios do Estado de São Paulo segundo a implantação do Saúde da Família. Rev Esc Enferm USP 2005; 39:28-35.

31. Guimarães TMR, Alves JGB, Tavares MMF. Impacto das ações de imunização pelo Programa Saúde da Família na mortalidade infantil por doenças evitáveis em Olinda, Pernambuco, Brasil. Cad Saúde Pública 2009; 25:868-76.

32. Oliveira SN, Silva SHD, Lunardelo SR. O Programa Saúde da Família como alternativa de mudança: um relato do município de Franca (SP). Investigação 2008; 8:115-20.

33. França ISX, Simplício DN, Alves FP, Brito VRS. Cobertura vacinal e mortalidade infantil em Campina Grande, PB, Brasil. Rev Bras Enferm 2009; 62: 258-64.

34. Ramos CF, Paixão JGM, Donza FCS, Silva AMP, Caçador DF, Dias VDV, et al. Cumprimento do calendário de vacinação de crianças em uma unidade de saúde da família. Revista Pan-Amazônica de Saúde 2010; 1:55-60.

35. Rodrígues G, Los Ángeles M. Magnitud y causas de oportunidades perdidas en vacunación en población menor de dos años en América. CES Med 2001; 15:71-80.

36. Chor D, Lima CRA. Aspectos epidemiológicos das desigualdades raciais em saúde no Brasil. Cad Saúde Pública 2005; 21:1586-94.

37. Willeman EM, Lima GR. O preconceito e a discriminação racial nas religiões de matriz africana no Brasil. Revista Uniabeu 2010; 3:70-94.

38. Fierman AH, Dreyer BP, Quinn L, Shulman S, Courtlandt CD, Guzzo R. Growth delay in homeless children. Pediatrics 1991; 88:918-25.

39. Jongpiputvanich S, Poomsuwan P, Phittayanon P. Prevalence and risk factors of protein energy malnutrition (PEM) in preschool children of KlongToey slum, Bangkok, Thailand. J Med Assoc Thai 1992; 75:39-45.

40. Fernandes BS, Jerônimo ML, Leone C. Características familiares e cuidados e condições de saúde das crianças: seu papel no risco de desnutrição protéico-calórica. Pediatria (São Paulo) 1996; 18:65-74.

41. Hirakata VN. Alternativas de análise para um desfecho binário em estudos transversais e longitudinais [Dissertação de Mestrado]. Pelotas: Universidade Federal de Pelotas; 1999.

Recebido em 01/Ago/2012

Versão final reapresentada em 07/Nov/2012 Aprovado em 13/Nov/2012 